\title{
TRAFFIC NOISE AND ITS EFFECT ON THE COMMUNITY
}

Amaa AbouEINour, H. A Zaffan, S. A Abouel-Seoud

\begin{abstract}
Noise at present day is now being regarded as one of the main pollutants to the human environment-life in the civilized society, so far has been dictated mainly by economic considerations and it is gratifying to note that lov $>$ s are being introduced to protect the community from unnecessary noise. However, the aim of the present paper is to discuss the road vehicle noise and its influence on community. The noise recordings are in terms of sound pressure level (SPL) and used to judge the actual traffic noise, repi isents a wide range of traffic conditions. It is stressed that criteria should be established in simple basic units and dBA values were recommended. By far, the most common single source of annoyance is that the road transportation noise and this aspect have been covered in specific detail. The results indicate that the averages SPL belong to the cross road locations perform the highest values despite of the type street route. All the measured values for all the streets routes considered are higher than those of the standard levels, where the residential streets route perform the highest level followed by the secondary streets route with the lowest levels for the main streets route.
\end{abstract}

